\title{
Urban air pollution by odor sources: short time prediction
}

\author{
Nicola Pettarin ${ }^{\mathrm{b}, \mathrm{c}}$, Marina Campolo ${ }^{1}$ and $^{\mathrm{a}}$, Alfredo Soldati ${ }^{\mathrm{b}, \mathrm{c}}$ \\ ${ }^{a}$ Dept. Chemistry, Physics $\&$ Environment, \\ University of Udine, 33100 Udine, Italy \\ ${ }^{b}$ Dept. Management, Electric $\&$ Mechanical Engineering, \\ University of Udine, 33100 Udine, Italy \\ ${ }^{c}$ LOD srl, 33100 Udine, Italy
}

\begin{abstract}
A numerical approach is proposed to predict the short time dispersion of odors in the urban environment. The model is based on (i) a three dimensional computational domain describing the urban topography at fine spatial scale (one meter) and on (ii) highly time resolved (one minute frequency) meteorological data used as inflow conditions. The time dependent, three dimensional wind velocity field is reconstructed in the Eulerian framework using a fast response finite volume solver of Navier-Stokes equations. Odor dispersion is calculated using a Lagrangian approach. An application of the model to the historic city of Verona (Italy) is presented. Results confirm that this type of odor dispersion simulations can be used (i) to assess the impact of odor emissions in urban areas and (ii) to evaluate the potential mitigation produced by odor abatement systems.
\end{abstract}

Keywords: Dispersion modelling, short averaging time, odor pollution,

\footnotetext{
${ }^{1}$ Corresponding author. Address via Cotonificio 108, 33100 Udine (UD), Italy; e-mail marina.campolo@uniud.it; phone: +39 432 558822; facs: +39 432558803
} 


\section{Introduction}

Exposure to unpleasant odors is one of the most frequent causes of air quality complaints in both industrial and urban areas. The chemical compounds responsible for odor generation are volatile species (Olafsdottir and Gardarsson, 2013): once emitted from a source, their transport, dispersion and fate in the environment is controlled by the complex interaction among strength of emission (Campolo et al., 2005), meteorological conditions and site topography. Odors become perceptible whenever the instantaneous and local concentration of these chemicals transpasses very low concentration values corresponding to the odor detection threshold. This may occur nearby a source but also some distance away from it.

Odor perception is synchronous with breathing and involuntary, but the subsequent reaction to a given odor stimulus is to some degree subjective: it depends on odor intensity and offensiveness, duration and frequency of exposure but also on pleasantness/unpleasantness of the sensation evoked by the odor (Blanes-Vidal et al., 2012). Annoyance may be produced from acute exposure to few, high odor intensity events or to chronic exposure to repeated, low odor intensity events (Griffiths, 2014). Whichever the exposure mode, odors generating a negative appraisal induce changes in people behavior and may trigger a stress-mediated response which may develop into a public health concern. Bad smells which occasionally cause annoyance, are proactively reported to the Health Services: when the source of the odor can be clearly identified and associated to a specific emission either by the 
analysis of resident nuisance odor reports (Nicolas et al., 2011), by the use of chemical sensors (Sohn et al., 2009; Seo et al., 2011) or by other sensory methods (Brattoli et al., 2011, Capelli et al., 2011), corrective actions can be devised as needed to contain/reduce the odor impact.

Odor impact in urban areas can be very difficult to assess and control due to the inherent complexity of the urban environment, the large number of potential sources and the local small scale variability of the dispersing wind. Odor nuisance is most frequently associated with discontinuous emissions generated by restaurants, fast food and bar which may occur for short/prolonged times (from a few seconds to minutes), occasionally or on a repetitive basis depending on the actual operating hours of the facility. The odor impact potentially arising from these commercial activities should be taken into account when planning new installations: best practices for design and operation of commercial kitchen ventilation systems have been developed (see DEFRA, 2005) and yet more accurate modelling tools could be profitably used for odour pollution assessment, prevention and mitigation. Odor emissions in a high populated urban area could be confidently authorized if the potential impact of each source could be estimated a priori by modelling; moreover, the precise evaluation of the odour impact of an existing source might be required for the detailed analysis of resident nuisance odor reports in support of litigations for odor impact problems.

Odor impact assessment based on chemical sensors would require the acquisition of highly time resolved, compound specific, qualified low-concentration data which are very difficult to obtain experimentally. Furthermore, most odors are generated by mixtures of compounds and the relationship between 
species concentration and odor nuisance is not straightforward. A more practical and effective approach may be the numerical prediction of odor dispersion.

Numerical models have been successfully used to predict odor dispersion and to assess odor impact in industrial areas (see Nicell, 2009, Sironi et al., 2010). The common approach is to model the odor as a passive chemical, equivalent to the mixture of chemicals present, whose concentration is conveniently represented by the number of odor units, a multiple of the mixture detection threshold. Most of the models in use has been adapted from earlier studies on air pollution: steady state Gaussian plume models (Latos et al., 2011), fluctuating plume models (Mussio et al., 2001; Dourado et al., 2014) and Lagrangian stochastic dispersion models (Franzese, 2003) have been used. The main challenge when using these models to predict odor dispersion is related with the different time and space resolution at which the prediction is required. The time scale of few seconds (corresponding to a single human breath) required to evaluate odor impact is much smaller than the hourly time scale typically used to evaluate the dispersion of pollutant species. If a hourly time scale is maintained for odor dispersion modelling, the peak odor concentration at the time scale relevant for odor impact assessment should be estimated using a peak to mean ratio, which can be either assumed to be constant (Sironi et al., 2010) or calculated based on wind speed, atmospheric stability, distance from and geometry of the source (Piringer et al., 2012; Schauberger et al., 2012).

Very different regulation limits and guidelines have been used worldwide to fix benchmark concentration for odors: Nicell (2009) reports values of off- 
site odor limits ranging from 0.5 to 50 odor units, averaging time ranging from $1 \mathrm{~s}$ to $1 \mathrm{hr}$ and compliance frequency ranging from $98 \%$ to $100 \%$. In Australia odor criteria (based on 3 minute average and 99.9\% frequency) are population density dependent (see EPA 373/07). The large variability in odor exposure criteria indicates that there is still little consensus on what odor concentration and/or averaging time represent the most effective and fair odor limits for off-site impact. Recently, odor criteria have been classified into two groups (Sommer-Quabach et al., 2014): those based on low odor concentration threshold and high exceedance frequency, relevant to assess chronic exposure, and those based on high concentration threshold and low exceedance frequency, relevant to assess acute exposure. At now, the recommended approach for odor regulation in Europe belongs to the first type (chronic exposure oriented) and consists in predicting by numerical models the hourly mean of odor concentration for at least one year period (up to 3 or 5 years) and to check odor exposure considering the 98th percentile of those data (see Environment Agency, 2011). The choice of the 98th percentile is supported by the strong correlation found with annoyance measured by community surveys (see Pullen and Vawda, 2007). Yet, different assessment tools and regulatory responses may be required to effectively manage acute exposure scenario (Griffiths, 2014).

A possibility is to use a smaller time scale for the odor dispersion modelling by which the peaks in odor concentration which result in annoyance for the population can be directly captured: Drew et al. (2007) demonstrated that dispersion modelling based on short averaging time was more successful than the current regulatory method at capturing odor peak con- 
centrations from a landfill site. Peak odor intensity is often associated with relatively weak meteorological dynamics (light winds) for which short term and short range effects may be important: wind directions can be highly variable (Huiling-cui et al., 2011), turbulent motions may be of the same order as wind speed and the shear production term may dominate in the turbulent kinetic energy budget equation (Manor, 2014) making the turbulent transport of species more sensitive to the presence of boundaries (complex terrain and presence of buildings) and highly anisotropic (Pitton et al., 2012).

Eulerian-Eulerian models based on Reynolds Averaged Navier Stokes (RANS) equations and Large Eddy Simulation (LES) have proven to be accurate to simulate the dispersion of chemical species (pollutants) in complex three dimensional domains (Hanna et al., 2006). Gailis et al. (2007) investigated tracer dispersion in a boundary layer sheared by a large array of obstacles using a Lagrangian stochastic plume model. They found that internal plume fluctuations can have a greater effect on tracer dispersion than the meander motion of the plume, which may be significantly damped in a roughwalled boundary layer. Michioka et al. (2013) implemented a short term, highly resolved (10 s) microscale large-eddy simulation (LES) model coupled to a mesoscale LES model to estimate the concentration of a tracer gas in an urban district considering both the influence of meteorological variability and topographic effects. Their results underlined the key role of coupling between mesoscale and local atmospheric dynamics in driving the dispersion of tracer gas.

The same type of short term, fine scale models can be used to simulate odor dispersion in the urban environment. Odor dispersion under steady 
wind and constant emission in the presence of few buildings has been evaluated using Eulerian-Eulerian Re-Normalisation Group (RNG) $k-\epsilon$ model by Maizi et al. (2010), using Large Eddy Simulation (LES) by Dourado et al. (2012) and using a fluctuating plume model by Dourado et al. (2014). Despite the increasing number of applications based on local, short averaging time dispersion models, this modelling approach has not yet been adequately validated to be confidently used for odor impact assessment (Pullen and Vawda, 2007). Moreover, we are not aware of applications of odor dispersion models to more complex urban environments. One of the reason is most likely the high cost associated with the time-dependent, fine resolved calculations needed to characterize the flow field of the carrier fluid and the transport of dispersed species into a complex domain. A fine spatial grid resolution (order of 1-2 meters) is required to model faithfully the complex urban domain and a fine time resolution is required to model concentration fluctuations and to capture the peak values responsible of the impact (see Pullen and Vawda, 2007). The simulation of local atmospheric dynamics highly resolved in space and time may become cheaper if representative scenarios rather than full year periods can be identified and considered. Moreover, cost/time of computation can be reduced adopting fast response models of Eulerian-Lagrangian type developed and used successfully to calculate dispersion of species in urban environments (Gowardhan et al., 2011).

In this work we propose the use of one of these models (QUIC - Quic Urban \& Industrial Complex model, Los Alamos Laboratories) (Gowardhan et al., 2011) to evaluate the impact of odor emissions in urban environments. The work is based on the assumption that the local wind field and turbulence 
controlling dispersion is triggered by the urban geometry more than by the microscale wind and atmospheric turbulence. Our objective is to demonstrate how different can be the odor impact evaluated in the short term when the dynamic interaction between wind field and complex urban topography is accounted for. We will use highly resolved (one minute frequency) microscale wind velocity data to reconstruct the flow field around buildings; this flow field will then be used to simulate the transport of odor, to evaluate odor exposure in terms of frequency of exceedance and intensity and to assess the potential odor impact. To demonstrate our idea, two different meteorological scenarios will be considered. Increasing the number of simulated scenarios enough to cover all the meteorological conditions that may influence the impact, the model could become a powerful tool to help Public Authorities in their planning and control activities.

First, we will to demonstrate that the proposed model can be used to evaluate comparatively the odor impact of a given emission source when located in alternative positions inside the urban micro-environment; second, we will prove that the model can be used to check if the odor impact can be sufficiently abated by the installation of odor control systems. The potential of the model will be demonstrated comparing the effect of untreated/treated emission associated to the planned installation of fast food activities in two different urban zones in the historical city of Verona (Italy). 


\section{Methods, site and data}

\subsection{Numerical model}

The model proposed (QUIC) is a 3D finite volume solver of ReynoldsAveraged Navier-Stokes (RANS) equations for incompressible flow. The model is implemented and runs in the Matlab environment. The computational domain, corresponding to an urban area including a large number of buildings, is defined using a structured grid in which solid/fluid cells are identified using numerical coding (zero and one identify solid and fluid cells, respectively). The grid is generated from Environmental Systems Research Institute (ESRI) shape files using the code built-in pre-processor.

RANS equations are solved explicitly in time on a staggered mesh using a projection method. The discretization scheme is second order accurate in space and time (see Gowardhan et al., 2011 for further details). A zero equation (algebraic) turbulence model is used. Free slip conditions are used at the top and side boundaries of the computational domain; a prescribed, time dependent velocity profile derived from an urban meteorological station can be imposed at the upwind side while an outflow boundary condition is imposed at the downwind side.

A Lagrangian particle approach is used to model odor dispersion: thousands of "particles" released from the emission point are tracked as they are randomly advected and dispersed over the domain (Zwack et al., 2011). Particles are modeled as infinitesimally small, neutrally buoyant gas parcels. For the present application, a steady state emission is considered for the odor plume: each particle is associated with a fraction of the odor emission rate and is tracked using a small time step (0.1 seconds). Overall, about half 
a million QUIC particles were released over the simulation time period (15 minutes).

Odor concentrations are determined in the Eulerian reference frame by counting how many particles pass through a given computational volume during the time averaging period of interest (30 seconds in our demo).

\subsection{Urban district}

Figure 1 (a) shows an aerial view of Verona downtown (near to the Arena). Two different zones were selected for modelling odor dispersion to check whether the specific localization of the source could significantly affect odor impact: the first area $(230 \times 290 \mathrm{~m}$ wide), identified as Area 1 , is characterized by street canyons; the second area $(495 \times 250 \mathrm{~m}$ wide), identified as Area 2, faces the open square of the Arena. Figures 1 (b) and (c) show the two computational models which extend $50 \mathrm{~m}$ above the ground.

The potential positions of the odor emission source in Area 1 and Area 2 are shown as red (light gray) dots $S 1$ and $S 2$. The emission height was fixed as one meter above the roof level. In the local coordinates system, with the grid origin at the lower left corner of each area, source positions are identified by $(x, y, z)$ triples equal to $(138.5,176.5,18.5)$ for Area 1 and $(161.5,238.5,17.5)$ for Area 2 . The blue (dark gray) circles indicate control points $P_{1}$ and $P_{2}$ located $50 \mathrm{~m}$ downstream the source in the prevailing wind blowing direction. The elevation of control points is $1.5 \mathrm{~m}$ above the ground.

\subsection{Meteorological data}

Data used in this work are taken from the urban station of Verona Golosine (latitude $45^{\circ} 28^{\prime} 51^{\prime \prime}$, longitude $10^{\circ} 52^{\prime} 35^{\prime \prime}, 61 \mathrm{~m}$ above sea level). One- 
minute time resolved records of wind speed and direction collected during February 2012 were made available from MeteoVerona. One week of data was statistically analysed. Statistics suggest that the prevailing wind blowing direction is from Nord, North-East (N-E) and the average wind speed is about $0.89 \mathrm{~m} / \mathrm{s}$ at the wind monitoring station (10 m elevation above the ground). To demonstrate how different can be the odor impact evaluated in the short term when time dependent winds interact with a complex urban topography, two 15 minute long periods were extracted for modelling odor dispersion: the first, event 1 , is characterized by wind intensity of $3.12 \pm 0.67 \mathrm{~m} / \mathrm{s}$ (average plus standard deviation) and wind blowing from direction $48 \pm 44^{\circ}$ degrees $\mathrm{N}$ (average plus standard deviation); the second, event 2, is characterized by wind intensity of $3.3 \pm 1.2 \mathrm{~m} / \mathrm{s}$ and wind blowing from direction $2 \pm 20^{\circ}$ degrees N. Even if average wind intensity is similar, variability of wind intensity is larger for event 2, whereas wind directions differ both in average value and variability. The two events selected are examples of "similar" and yet substantially different scenarios which need to be simulated to obtain a consistent evaluation of odor impact. Considering the size of computational domain and average wind intensity, each 15 minute long period is long enough to track the dispersion of the odor plume up to the boundaries of the computational domain. More/longer periods could be routinely simulated once extended meteorological data are made available.

Figure 2 shows the wind variation of the two selected events using a polar representation (Figure $2(\mathrm{a})$ ) and time series plots of wind speed and direction (Figure 2 (b) and (c)). At each time step, the direction from which the wind is blowing identifies the upstream side of the computational domain; 
the vertical profile of wind velocity used as inflow condition is defined by the wind speed recording at the anemometer (red arrow in Figure 1 (b)) using a power law.

\subsection{Emission data}

To characterize the strength of the emission, we considered a restaurant using the same cooking methods (deep frying and stewing) of the planned fast food installation. Samples used to quantify the odor emission rate were collected from the chimney of the restaurant when a frying food system was active. The mean cooking time for lunch (or dinner) period was 109 minutes. The stack diameter was $1 \mathrm{~m}$. The mean values of stack outlet velocity and exhaust flow rate were $4.12 \mathrm{~m} / \mathrm{s}$ and $350 \mathrm{Nm}^{3} / \mathrm{min}$. The mean stack inlet and outlet temperatures were $44^{\circ} \mathrm{C}$ and $31^{\circ} \mathrm{C}$. The variability of the source was checked during sampling according to EN ISO 16911:2013. We collected three samples according to EN 13725:2003 using a vacuum pump to suck air from the emitting stack into Nalophan bags (8 L volume); sampling required about 1.5 minutes for each sample, with 10 minute stop between samples to check emission variability over time; odor samples were then transferred to the lab for the sensory evaluation of odors off site by a group of trained panels. Mixtures of sampled air and neutral air at decreasing dilution ratio were sequentially prepared by the olfactometer and smelt by the panels. The test started from an odor sample which was very diluted. The dilution ratio was gradually reduced up to the identification of the odor threshold, i.e. the point at which the odor is only just detectable to $50 \%$ of the test panel. The numerical value of the dilution ratio necessary to reach the odor threshold was taken as the measure of the odor concentration at the source expressed 
in European odor units per cubic meter $\left(o . u \cdot E / m^{3}, o u / m^{3}\right.$ in brief $)$.

Sampling was performed in two different working conditions, corresponding to off/on operation for the activated carbon filter installed for odor control. Data collected during sampling are summarized in Table 1. Data variability during sampling and among samples was found to be not significant and odor emission rates used to set up the model are values averaged over the three samples.

\section{Results}

\subsection{Flow field}

The QUIC code calculates the flow field in the three dimensional domain every one minute. Figure 3 shows the comparison between the wind speed/direction measured at the meteorological station (10 m height, line with circles) and used as inflow condition, and those calculated in different points of the computational domain: at the source $(1 \mathrm{~m}$ above roof level, empty triangles) and at the reference control point (solid triangles) for Area 1 (triangles pointing upward) and Area 2 (triangles pointing downward) $(1.5 \mathrm{~m}$ above ground). The effect of urban topography is to produce local differences in wind intensity and direction calculated at different points.

Wind speed and direction calculated at the emission point (i.e. above the buildings) are similar to the values recorded at the meteorological station: the wind speed is a bit larger at the emission point since it is more elevated than the anemometric sensor. At control points, the wind speed is generally smaller than the sensor due to the different elevation above the ground $(1.5 \mathrm{~m})$; the wind direction may be significantly different. For control 
points located in a street canyon, the effect of the urban topography is to smooth out the variability of wind direction. For wind event 1, the local wind direction is about $50^{\circ} \mathrm{N}$ whichever the value recorded at the meteorological station for both control points $\mathrm{P} 1$ and $\mathrm{P} 2$; for event 2, the wind direction is similar at the meteorological station and point P2, whereas it is always about $50^{\circ} \mathrm{N}$ for point $\mathrm{P} 1$.

\subsection{Odor dispersion}

Animations of the odor plume dispersing from sources S1 and S2 during the two simulated wind events are available as supplementary material. The position of the emission point is indicated by the black circle; isocontours represent the odor concentration (in ou $/ \mathrm{m}^{3}$ ) calculated in the plane $1.5 \mathrm{~m}$ above the ground (reference height of human noses potentially smelling in the area). Figures 4-5 shows snapshots (one every 240 seconds) taken from the animations. The color scale for odor concentration shown in the plots in limited to the sub-range $\left[2 \div 12 \mathrm{ou} / \mathrm{m}^{3}\right]$. To relate odor concentration to perceived odor intensity in the field we refer to the following scale (SommerQuabach et al., 2014): non detectable $\left(C<2\right.$ ou $\left./ \mathrm{m}^{3}\right)$, acceptable $(2<$ $\left.C<5 \mathrm{ou} / \mathrm{m}^{3}\right)$, annoyance $\left(5<C<15 \mathrm{ou} / \mathrm{m}^{3}\right)$ and severe annoyance $\left(C>15 \mathrm{ou} / \mathrm{m}^{3}\right)$. The lower and upper values of the color scale represent an odor concentration threshold at which the odor is clearly detected and a value at which the odor perceived is strong enough to cause annoyance.

Isocontours calculated during wind event 1 in Area 1 (Figure 4 upper row) show the odor plume extending in different directions depending on the leading wind. Yet, the urban topography determines a preferential path for odor dispersion which spreads along the main street canyons near to the 
source. Due to the changing wind direction, some of the odor puffs may reach regions not directly exposed to the emitting source, producing diffuse odor impact even at significant distances. During wind event 2, isocontours (Figure 4 bottom row) show odor puffs moving along three main street canyons (aligned with the wind blowing directions) with odor concentration mainly controlled by wind speed. Odor dispersion produced in Area 2 (open area facing the Arena) for wind event 1 (Figure 5 top row) indicates that odor puffs remain confined along the prevailing wind direction (from N-E to SW) despite the wind direction variability, and may penetrate into the urban topography when the blowing wind direction is from S-E. For wind event 2 (Figure 5 bottom row) the odor plume oscillates back and forth in the open square facing the Arena.

The dynamic evolution of odor isocontours gives a qualitative idea of the odor impact expected from the emission, given the position and the meteorological scenario. Yet, for a quantitative comparison we need more synthetic descriptors which can be obtained from the statistical analysis of the time series of odor concentration calculated for each grid point of the computational domain.

Figure 6 shows the time series of odor concentration calculated during wind event 1 for the grid point closest to the emission source S1 and for point P1. According to the FIDOL methodology (see Environment Agency, 2011) the intensity and frequency of odor exposure are two of the main characteristics necessary to assess the offensiveness of odors. Due to the short averaging time and brief simulation period used in this work we can not use the recommended regulation approach to assess odor impact. We propose to 
use two odor impact criteria similar to those discussed by Griffiths (2014), based on either intensity or frequency of odor impact events evaluated over the time interval of interest (the 15 minute long period in our case). Specifically, for the first odor criteria, we fix the frequency of exceedance (10\%) and derive odor concentration isocontours which can be compared against threshold values; for the second odor criteria, we fix an odor concentration threshold $\left(C_{r e f}=5 \mathrm{ou} / \mathrm{m}^{3}\right)$ and derive maps of frequency of exceedance. Any specific value of frequency of exceedance and odor concentration threshold could be adopted to perform the kind of analysis we propose.

Figure 6 shows that near to the source (S1) the odor concentration is quite large $\left(653 \pm 100 \mathrm{ou} / \mathrm{m}^{3}\right.$ average value \pm standard deviation, coefficient of variation equal to 0.15 ) and only slightly changing over time; at point $\mathrm{P} 1$, the odor intensity is significantly lower $\left(11 \pm 8.7 \mathrm{ou} / \mathrm{m}^{3}\right.$ average value \pm standard deviation, coefficient of variation equal to 0.79) but the variability in time is larger. The $90^{t h}$ percentiles are equal to 24.4 (indicated as dashed thin line in the graph) and $802.6 \mathrm{ou} / \mathrm{m}^{3}$ (not shown) for P1 and $\mathrm{S} 1$; the reference threshold concentration $C_{r e f}$ (dashed thick line in the graph) is exceeded $80 \%$ of time at P1 and $100 \%$ of time at S1.

Figure 7 shows the results of this analysis replicated for each point of the computational grid: this can be used to compare and rank, according to the two proposed assessment criteria, the odor impacts for Area 1 and Area 2 for simulated wind events. Isocontours of $90^{\text {th }}$ percentile of odor concentration are shown in the top row and isocontours of the exceedance frequency $\left(C>C_{r e f}\right)$ are shown in the bottom row. These maps show the area in which any plotted concentration of odor is exceeded $10 \%$ of the 
time at maximum, or the area in which detectable odor may be perceived persistently (i.e. most frequently) in time.

Comparison between isocontours of $90^{\text {th }}$ percentile calculated for Area 1 and Area 2 for wind event 1 (Figure 7, left half) indicates that the emission will produce annoyance/severe annoyance at least $10 \%$ of the time along the main street canyon in Area 1 and in front of the buildings facing the Arena in Area 2. Detectable odor will be perceived for more than $50 \%$ of the time in these areas.

The odor impact becomes even more significant for wind event 2 (Figure 7 , right half). In this case, the emission will produce annoyance/severe annoyance at least $10 \%$ of the time along the three street canyons for Area 1 and in a wide area close to the Arena in Area 2. Detactable odor will be perceived for more than $50 \%$ of the time in even wider areas.

Figure 8 shows a final synthetic picture of odor impact given in the form of odor roses, i.e. polar plots in which (i) the $90^{t h}$ percentile of odor concentration (top half) or (ii) the percent frequency of exceedance of $C_{r e f}$ (bottom half) are plotted at reference distances (5, 25 and $45 \mathrm{~m}$ away from the emission point) for each angular direction. Top and bottom rows in each half represent the impact of the emission as is (untreated) or when the odor abatement system is on. The radial scale of each plot is shown in the bottom right corner. Consider first the impact of untreated source, S1 and S2, for wind event 1 (first row, left half). The peak of odor concentration is found in the south-west $(\mathrm{S}-\mathrm{W})$ direction, with odor concentrations as large as $20 \mathrm{ou} / \mathrm{m}^{3}$ 25 and $45 \mathrm{~m}$ away from emission point $\mathrm{S} 1$ and as large as $40 \mathrm{ou} / \mathrm{m}^{3} 25$ and $45 \mathrm{~m}$ away from emission point S2. Minor peaks are also found along those 
directions in which the wind and the local topography are "in phase". The frequency of exceedance of $C_{r e f}$ (third row, left half) is up to $60 \%$ both 25 and $45 \mathrm{~m}$ away from emission point $\mathrm{S} 1$ in the $\mathrm{S}-\mathrm{W}$ direction, and up to $55 \%$ and $75 \%$ respectively 25 and $45 \mathrm{~m}$ away from emission point $\mathrm{S} 2$ in the same direction. When the abatement system is on (second and fourth rows), the odor impact becomes lower than $10 \mathrm{ou} / \mathrm{m}^{3}$ whichever the distance and angular direction and $C_{r e f}$ is exceeded $50 \%$ of the time at most. The right half of Figure 8 shows the odor impact for wind event 2. In this case, the peak of odor concentration is in the south-south-west (S-S-W) direction, with odor concentrations as large as 40 and $76 \mathrm{ou} / \mathrm{m}^{3}$ respectively $5 \mathrm{~m}$ and $25 \mathrm{~m}$ away from emission point S2. The frequency of exceedance is about $100 \% 25 \mathrm{~m}$ and $45 \mathrm{~m}$ away from $\mathrm{S} 2$ in the $\mathrm{S}-\mathrm{W}$ direction. These data indicate a more intense and persistent odor impact for wind event 2. The odor impact can be reduced in Area 1 treating the emission (second and fourth rows), with annoying odor perceived less than $40 \%$ of the time $25 \mathrm{~m}$ away from the source in the S-W direction. Annoying odor can be still perceived up to $60 \%$ of the time $45 \mathrm{~m}$ away from the source in the $\mathrm{W}-\mathrm{S}-\mathrm{W}$ direction. The situation is more critical for the source located in Area 2: even if the abatement system reduces the odor impact, annoying odor will still be perceived $80 \%$ of the time in the $\mathrm{S}-\mathrm{W}$ direction 25 and $45 \mathrm{~m}$ away from the source.

\section{Conclusions}

In this work we propose the use of a fast response Eulerian-Lagrangian type model to calculate the short term, short time average dispersion of odor in urban areas. The model is based on a three dimensional computational 
domain describing the urban topography at fine (one meter) spatial scale and on highly time resolved (one minute frequency) meteorological data used as inflow conditions.

We propose two odor impact criteria similar to those discussed by Griffiths (2014) to assess odor impact: for the first odor criteria we fix the frequency of exceedance $(10 \%)$ to derive odor concentration isocontours which can be compared against threshold values; for the second odor criteria we fix an odor concentration threshold $\left(C_{r e f}=5 \mathrm{ou} / \mathrm{m}^{3}\right)$ to derive maps of frequency of exceedance. Simulations performed for the historical city of Verona for two 15 minute long time periods show that the model can be used (i) to comparatively evaluate and rank the odor impact of a given emission source when located in alternative positions of the urban area; (ii) to check if end of pipe technologies devised for odor control are effective or not to reduce the impact.

We propose the odor rose plot of model output statistics $\left(90^{\text {th }}\right.$ percentile and exceedance frequency) as a simple graphical tool to compare odor impact for different source locations and in different meteorological scenarios and to evaluate the effectiveness of solutions proposed for odor impact mitigation.

\section{Acknowledgements}

The authors would like to thank Comune di Verona for geographical data and R. Snidar and S. Rivilli for fruitful discussions. M.C. gratefully acknowledges Michael Brown and R\&D staff of Los Alamos National Laboratory for the use of QUIC code and technical support, and MeteoVerona for the acquisition of highly resolved wind data. N.P. gratefully acknowledges Laboratorio 
di Olfattometria Dinamica, Udine, Italy for funding his PhD fellowship.

[1] Blanes-Vidal, V., Suh, H., Nadimi, E.S., Løfstrøm, P., Ellermann, T., Andersen, E.V., Schwartz, J., 2012, Residential exposure to outdoor air pollution from livestock operations and perceived annoyance among citizens, Environment International, 40: 44-50.

[2] Brattoli, M., de Gennaro, G., de Pinto, V., Loiotile, A.D., Lovascio, S., Penza, M., 2011, Odour Detection Methods: Olfactometry and Chemical Sensors, Sensors, 11(5): 5290-5322.

[3] Campolo, M., Salvetti, M.V., Soldati, A., 2005, Mechanisms for microparticle dispersion in a jet in crossflow, AICHE J., 51(1): 28-43.

[4] Capelli, L., Sironi, S., Del Rosso, R., Centola, P., Rossi, A., Austeri, C., 2011, Olfactometric approach for the evaluation of citizens' exposure to industrial emissions in the city of Terni, Italy, Science of the Total Environment, 409: 595-603.

[5] DEFRA, Department for Environment, Food and Rural Affairs 2005, Guidance on the Control of Odour and Noise from Commercial Kitchen Exhaust Systems, Nobel House, 17 Smith Square, London SW1P 3JR.

[6] Dourado, H., Santos, J.M., Reis, N.C., Mavroidis, I., 2012, Numerical modelling of odour dispersion around a cubical obstacle using large eddy simulation, Water Science and Technology, 66(7): 1549-1557.

[7] Dourado, H., Santos, J.M., Reis, N.C., Mavroidis, I., 2014, Development of a fluctuating plume model for odour dispersion around buildings, Atmospheric Environment, 89: 148-157. 
[8] Drew, G.H., Smith, R., Gerard, V., Burge, C., Lowe, M., Kinnersley, R., Sneath, R., Longhurst, P.J., 2007, Appropriateness of selecting different averaging times for modelling chronic and acute exposure to environmental odours, Atmospheric Environment, 41(13): 2870-2880.

[9] EN 13725:2003, Air quality - Determination of odour concentration by dynamic olfactometry.

[10] EN ISO 16911:2013, Stationary source emissions - Manual and automatic determination of velocity and volume flow rate in ducts - Part 1: Manual reference method.

[11] Environment Agency, 2011, Additional guidance for H4 Odor Management: How to comply with your environmental permit, Environment Agency Horizon House, Deanery Road Bristol, BS1 5AH.

[12] EPA 373/07 guidelines: Odour assessment using odour source modelling, 2007, www.epa.sa.gov.au/files/477172_guide_odour.pdf

[13] Franzese, P., 2003, Lagrangian stochastic modeling of a fluctuating plume in the convective boundary layer, Atmospheric Environment, 37(12): 1691-1701.

[14] Gailis, R.M., Hill, A., Yee, E., Hilderman, T., 2007, Extension of a fluctuating plume model of tracer dispersion to a sheared boundary layer and to a large array of obstacles, Boundary-Layer Meteorol., 122: 577607.

[15] Gowardhan, A.A., Pardyjak, E. R., Senocak, I., Browm, M.J., 2011, A 
CFD-based wind solver for an urban fast response transport and dispersion model, Environmental Fluid Mechanics, 11(5): 439-464.

[16] Griffiths, K.D., 2014, Disentangling the frequency and intensity dimensions of nuisance odour, and implications for jurisdictional odour impact criteria, Atmospheric Environment, 90: 125-132.

[17] Hanna, S.R., Brown, M.J., Camell, F.E.; Chan, S.T., Coirier, W.J., Hansen, O.R., Huber, A.H., Kim, S., Reynolds, R.M., 2006, Detailed simulations of atmospheric flow and dispersion in downtown Manhattan: An application of five computational fluid dynamics models, Bulletin of the American Meteorological Society, 87(12): 1713-1726.

[18] Huiling cui, Yao, R., Xu, X., Xin, C., Yang, J., 2011, A tracer experiment study to evaluate the CALPUFF real time application in a near-field complex terrain setting, Atmospheric Environment, 45: 7525-7532.

[19] Latos, M., Karageorgos, P., Kalogerakis, N., Lazaridis, M., 2011, Dispersion of Odorous Gaseous Compounds Emitted from Wastewater Treatment Plants, Water Air Soil Pollut., 215: 667-677.

[20] Maizi, A., Dhaouadi, H., Bournot, P., Mhiri, H., 2010, CFD prediction of odorous compound dispersion: Case study examining a full scale waste water treatment plant, Biosystems Engineering, 106: 68-78.

[21] Manor, A., 2014, A Stochastic Single-Particle Lagrangian Model for the Concentration Fluctuations in a Plume Dispersing Inside an Urban Canopy, Boundary-Layer Meteorology, 150(2): 327-340. 
[22] Michioka, T., Sato, A., Sada, K., 2013, Large-eddy simulation coupled to mesoscale meteorological model for gas dispersion in an urban district, Atmospheric Environment, 75: 153-162.

[23] Mussio, P., Gnyp, A.W., Hensha, P.F., 2001, A fluctuating plume dispersion model for the prediction of odour-impact frequencies from continuous stationary sources, Atmospheric Environment, 35: 2955-2962.

[24] Nicell, J.A., 2009, Assessment and regulation of odour impacts, Atmospheric Environment, 43 (1): 196-206.

[25] Nicolas, J., Cors, M., Romain, A.C., Delva, J., 2011, Identification of odour sources in an industrial park from resident diaries statistics, Atmospheric Environment, 44(13): 1623-1631.

[26] Olafsdottir, S., Gardarsson, S.M., 2013, Impacts of meteorological factors on hydrogen sulfide concentration downwind of geothermal power plants, Atmospheric Environment, 77: 185-192.

[27] Piringer, M., Schauberger, G., Petz, E., Knauder, W., 2012, Comparison of two peak-to-mean approaches for use in odour dispersion models, Water Science and Technology, 66(7): 1498-1501.

[28] Pitton, E., Marchioli, C., Lavezzo, V., Soldati, A., Toschi, F., 2012, Anisotropy in pair dispersion of inertial particles in turbulent channel flow, Phys. Fluids, 24(7): 073305.

[29] Pullen, J., Vawda, Y., 2007, Review of Dispersion Modelling for Odour Predictions, Science Report: SC030170/SR3 Environment Agency, Rio House, Waterside Drive, Aztec West, Almondsbury, Bristol, BS32 4UD 
[30] Schauberger, G., Piringer, M., Schmitzer, R., Kamp, M., Sowa, A., Koch, R., Eckhof, W., Grimm, E., Kypke, J., Hartung, E., 2012, Concept to assess the human perception of odour by estimating short-time peak concentrations from one-hour mean values. Reply to a comment by Janicke et al., Atmospheric Environment, 54: 624-628.

[31] Sironi, S., Capelli, L., Centola, P., Del Rosso, R., Pierucci, S., 2010, Odour impact assessment by means of dynamic olfactometry, dispersion modelling and social participation, Atmospheric Environment, 44(3): 354-360.

[32] Sohn, J.H., Pioggia, G., Craig, I.P., Stuetz, R.M., Atzeni, M.G., 2009, Identifying major contributing sources to odour annoyance using a nonspecific gas sensor array, Biosystems Engineering, 102(3): 305-312.

[33] Sommer-Quabach, E., Piringer, M., Petz, E., Schauberger, G., 2014, Comparability of separation distances between odour sources and residential areas determined by various national odour impact criteria, Atmospheric Environment, 95: 20-28.

[34] Zwack, L.M., Hanna, S.R., Spengler, J.D., Levy, J.I., 2011, Using advanced dispersion models and mobile monitoring to characterize spatial patterns of ultrafine particles in an urban area, Atmospheric Environment, 45(28): 4822-4829. 
(a)

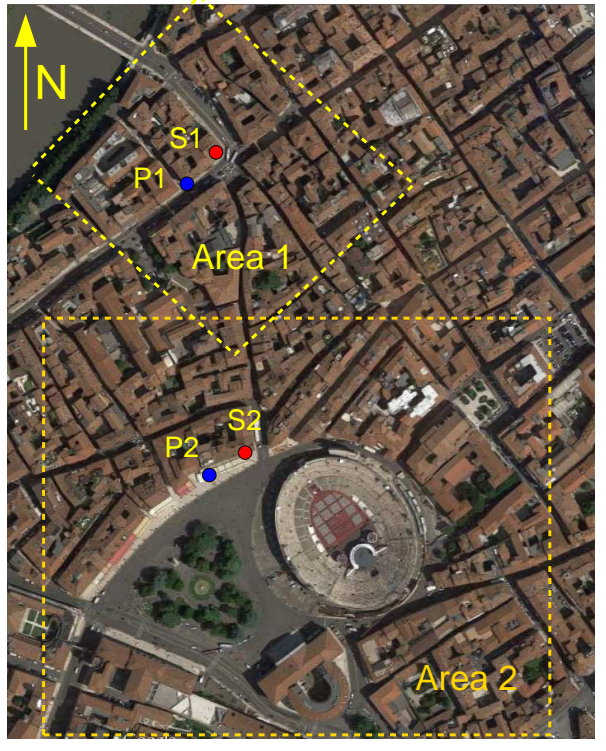

(b)

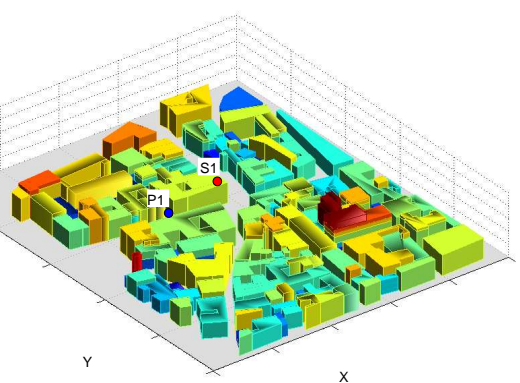

(c)

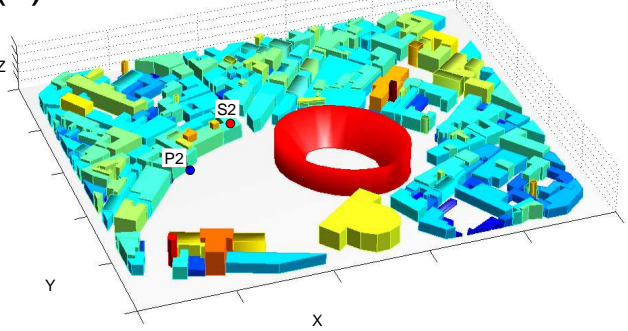

Figure 1: Aerial view of Verona (a) and areas selected for odor dispersion demo: (b) street canyons (Area 1) and (c) open square nearby the Arena (Area 2); potential positions of odor emission source are shown as (light gray) red circles (S1 and S2); points $50 \mathrm{~m}$ away from the source downstream the prevailing blowing wind direction (N-E) are shown as (dark gray) blue circles (P1 and P2). 
(a)

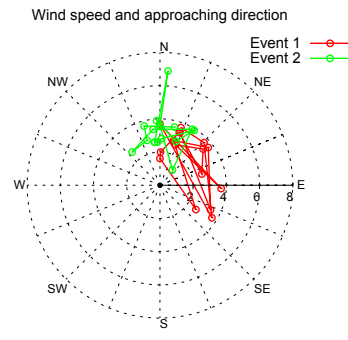

(b)

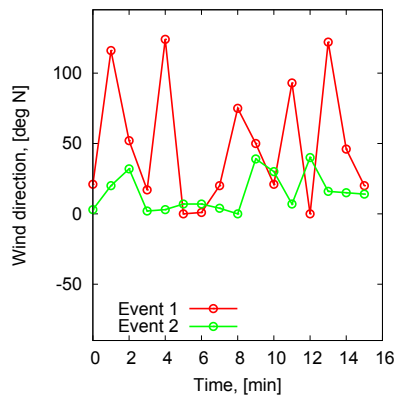

(c)

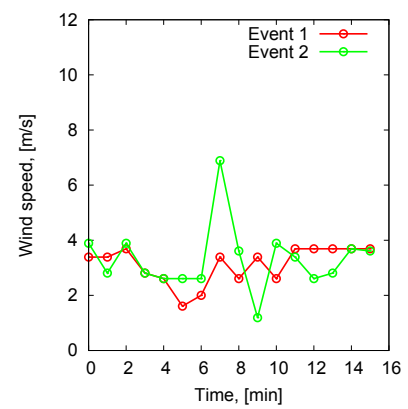

Figure 2: Polar representation (a) and time series plots of wind direction (b) and wind speed (c) of wind data extracted for simulating odor dispersion: data are taken from meteorological station of Verona Golosine. 
(a)

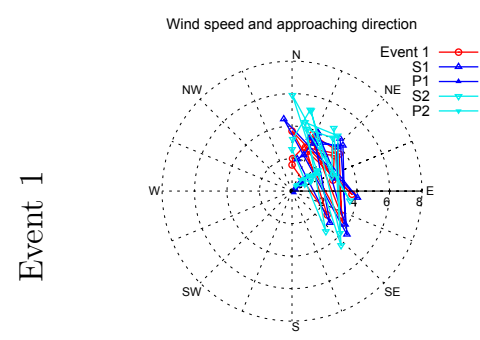

(d)

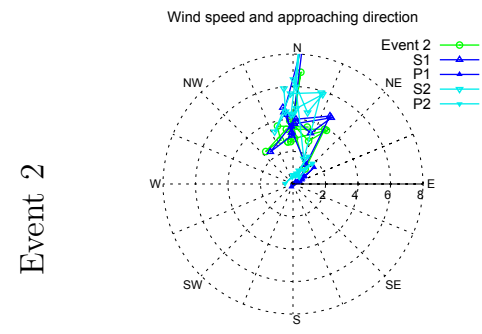

(b)

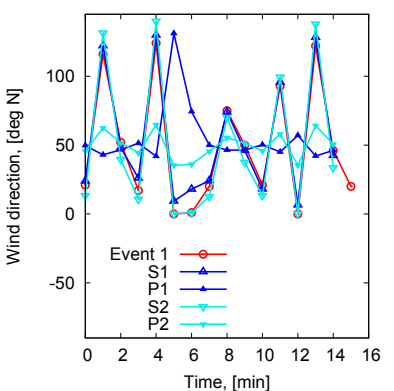

(e)

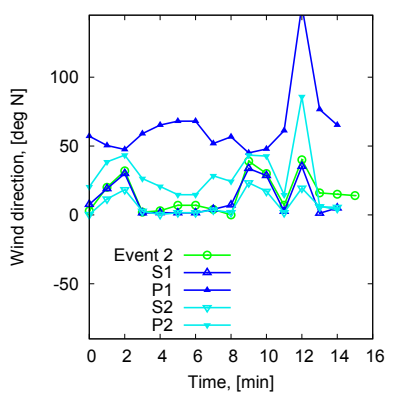

(c)

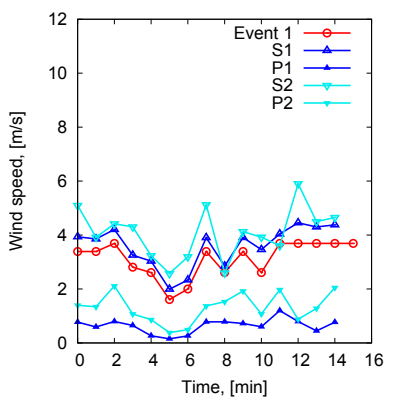

(f)

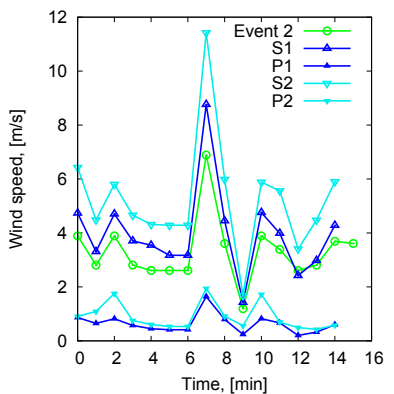

Figure 3: Polar representation (a, d) and time series plots of wind direction (b, e) and wind speed (c, f) calculated in different points of the computational domain for wind events 1 (top row) and 2 (bottom row): lines with symbols correspond to (i) anemometric data used as inflow condition (10 $\mathrm{m}$ above ground) (red/green, solid), (ii) emission point position (1 m above roof level) (solid symbol, S1 blue/dark gray, S2 pale blue/light gray), (iii) control point position (1.5 m above ground) (empty symbol, P1 blue/dark gray, P2 pale blue/light gray). 
Area 1

$240 \mathrm{~s}$

$480 \mathrm{~s}$

$720 \mathrm{~s}$
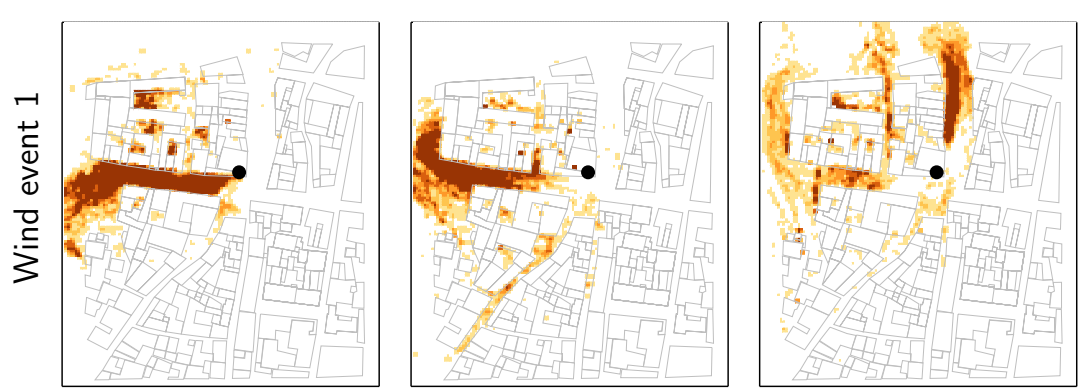

$\mathrm{ou} / \mathrm{m}^{3}$
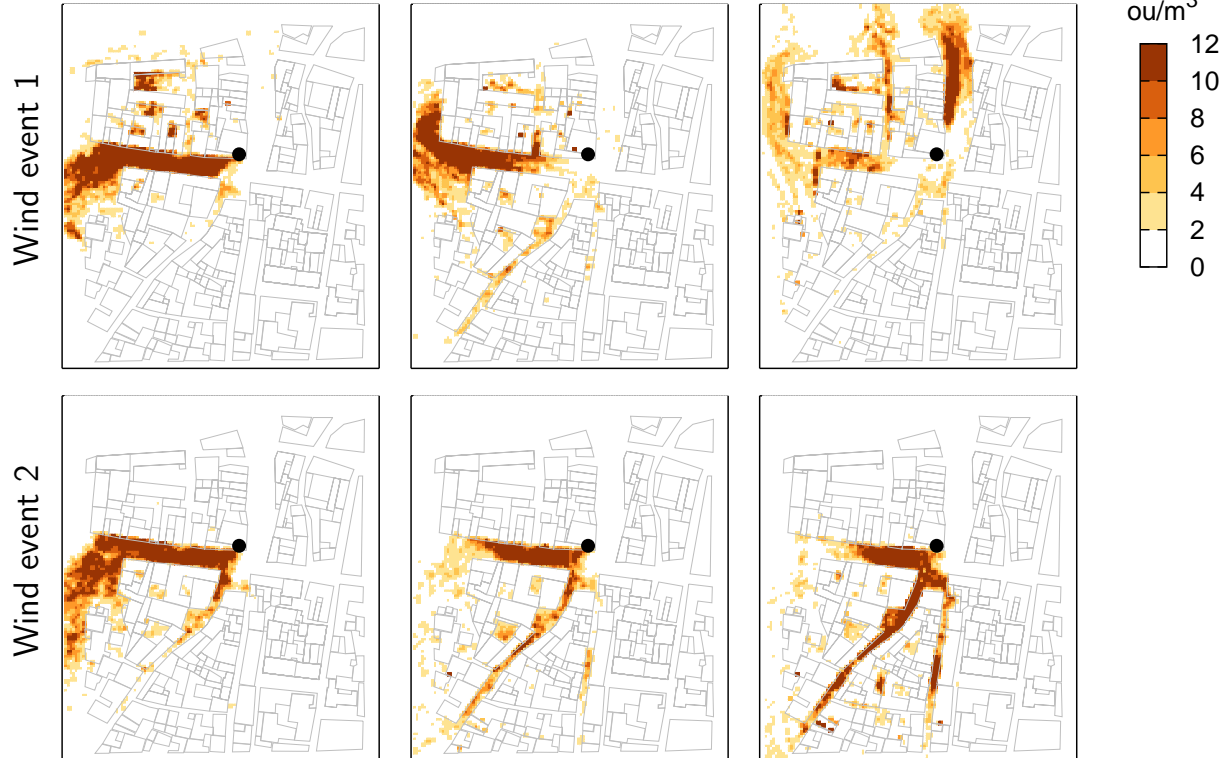

Figure 4: Isocontours of odor concentration calculated for Area 1 and wind event 1 and 2. Values are shown for a plane $z=1.5 \mathrm{~m}$ above the ground: snapshots are taken at every $240 \mathrm{~s}$. 
Area 2

$240 s$
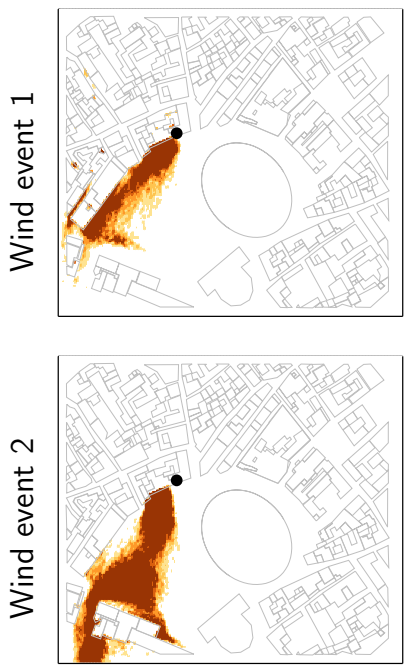

$480 \mathrm{~s}$


$720 \mathrm{~s}$
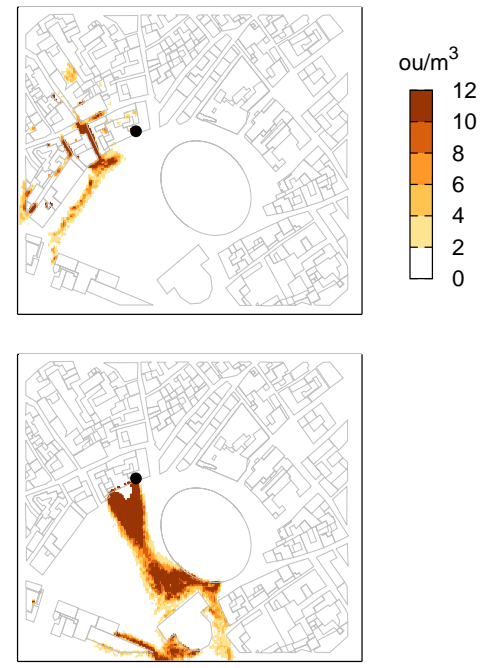

Figure 5: Isocontours of odor concentration calculated for Area 2 and wind event 1 and 2 . Values are shown for a plane $z=1.5 \mathrm{~m}$ above the ground: snapshots are taken at every $240 \mathrm{~s}$. 




Figure 6: Time series of 30 seconds average odor concentration calculated at point P1 (closed symbol) and S1 (open symbol) for wind event 1: dashed lines represent $90^{\text {th }}$ percentile of odor concentration for point P1 (thin dashed line) and a reference odor concentration threshold ( $5 \mathrm{ou} / \mathrm{m}^{3}$, thick dashed line) sufficient to cause nuisance. 
Wind event

Area 1

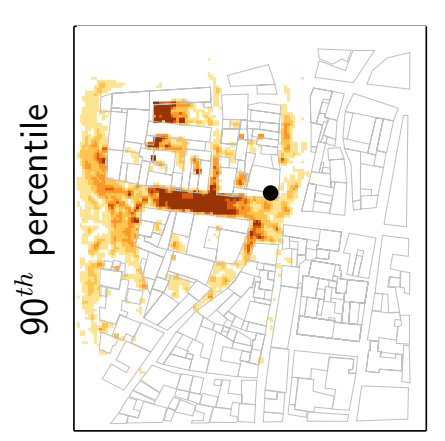

$\omega$



Area 2


Wind event 2

Area 1
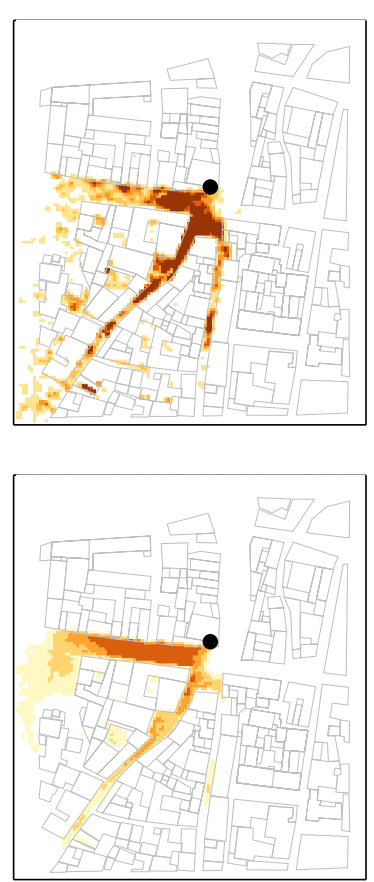

Area 2


\% Exceed

100
-80

- 60

40

$-20$

Figure 7: Statistics for odor impact assessment: (a) $90^{\text {th }}$ percentile of odor concentration and (b) percent of exceedances $(C>$ $5 \mathrm{ou} / \mathrm{m}^{3}$ ) during wind event 1 and 2 in Area 1 and 2. 




Figure 8: Odor rose of $90^{t h}$ percentile of odor concentration and \% Exceedances for untreated and treated emission S1 and S2 and wind event 1 and 2. 


\begin{tabular}{lllll}
\hline Sample N. & $\mathrm{T},\left[{ }^{\circ} \mathrm{C}\right]$ & $\mathrm{RH},[\%]$ & $\mathrm{Q},\left[\mathrm{Nm}^{3} / \mathrm{s}\right]$ & $\mathrm{C},\left[\mathrm{ou} / \mathrm{m}^{3}\right]$ \\
\hline $\mathrm{U}-1$ & 31.3 & 24.1 & 5.4 & 5,000 \\
$\mathrm{U}-2$ & 29.5 & 22.4 & 6.2 & 3,800 \\
U-3 & 32.2 & 28.7 & 5.9 & 5,000 \\
\hline Average & 31.0 & 25.07 & 5.83 & 4,600 \\
\hline T-1 & 30.8 & 24.6 & 6.0 & 1,300 \\
T-2 & 30.3 & 22.7 & 4.3 & 1,300 \\
T-3 & 30.9 & 23.5 & 4.5 & 2,000 \\
\hline Average & 30.7 & 23.6 & 4.93 & 1,533 \\
\hline
\end{tabular}

Table 1: Results of odor source sampling: $U$ (untreated) identifies odor emission with abatement system turned off, $T$ (treated) identifies odor emission with abatement system turned on. 